\title{
On Constructive Cut Admissibility in Deduction Modulo
}

\author{
Richard Bonichon ${ }^{1}$ and Olivier Hermant ${ }^{1}$ \\ Université Paris 6 - LIP6 *
}

\begin{abstract}
Deduction modulo is a theoretical framework which allows the introduction of computational steps in deductive systems. This approach is well suited to automated theorem proving. We describe a proofsearch method based upon tableaux for Gentzen's intuitionistic LJ extended with rewrite rules on propositions and terms . We prove its completeness with respect to Kripke structures. Then we give a soundness proof with respect to cut-free LJ modulo. This yields a constructive proof of semantic cut elimination, which we use to characterize the relation between tableaux methods and cut elimination in the intuitionistic case.
\end{abstract}

\section{Introduction}

The road to automated deduction has many pitfalls. Efficient treatment of equality and equational theories is for instance a challenging area and proving $(a+b)+((c+d)+e)=a+((b+c)+(d+e))$ with the usual associativity and identity axioms can easily lead to an infinite loop when using an ineffective strategy. What we would like is a deterministic and terminating method where we only have to check if the two terms are indeed the same modulo the given axioms. We would rather use computation (aka blind execution) instead of deduction (non-deterministic search), thus transforming the associativity axiom into a term-rewriting rule. Although the orientation of equational theories using rewriting techniques is nothing unusual, propositional rewrite rules are hardly considered in the literature. However it can be useful to allow them. One framework to handle such rewrite rules is deduction modulo ([4]). The rewrite rule $x * y=0 \rightarrow x=0 \vee y=0$ is the orientation of the axiom $\forall x \forall y \quad(x * y=0 \Leftrightarrow(x=0 \vee y=0))$ and can be used to prove $\exists z(a * a=z \Rightarrow a=z)$ by automated deduction methods ([1, 4]) adapted to use such rules. This rule can not be easily turned into a term-rewriting rule.

Using rewrite rules instead of unoriented axioms should speed up automated theorem provers. However, deduction modulo has other interesting consequences: propositional rewrite rules can be used to restart a deductive process such as in $P(a) \rightarrow \forall x P(x)$. Also, the proof skeleton of a proof modulo only contains the important deductive steps — or those a human sees as important — and not the computational details.

\footnotetext{
* Laboratoire d'Informatique de Paris 6, 8 rue du Capitaine Scott, 75015 Paris, France email: (richard.bonichon | olivier.hermant)@lip6.fr
} 
Classical first-order logic modulo has first been studied in [4], However, intuitionistic logic is particularly interesting as it has the witness and the disjunction properties, and is therefore adapted to constructive mathematics and computer science, through the Curry-Howard isomorphism. Intuitionistic automated deduction procedures seem less studied, maybe because intuitionistic semantics are harder to deal with. In particular, two main different semantics exist: Kripke structures and Heyting algebras.

In this paper, we focus on the cut-elimination theorem. Starting with Gentzen's result, it has turned out to be one of the most important properties in the field of logic. Cut-elimination in deduction modulo is a harder problem because this property is not valid even for all confluent and terminating rewrite systems. The result can be obtained in two different ways: the first is syntactic and proves termination of a certain cut-elimination process, its modern variant uses proof terms [5] and the reducibility method; the other proves the admissibility (or redundancy) of the cut rule by establishing the completeness of the cut-free calculus with respect to some notion of model. Known since Beth and Hintikka, the latter has been recently used by De Marco and Lipton [3].

This article shows the deep link between all these topics. First, we recall the intuitionistic sequent calculus modulo defined in [7] ( Sec. 2) and its semantics.

- We give a proof-search method for $L J_{\text {mod }}$ (Sec. 3 ). Such methods are often based on tableaux when dealing with nonclassical logics. We here formulate a tableau method modulo rewrite rules on terms and propositions and conditions on their use.

- We prove that this method enjoys the usual property of completeness in Sec. 4. We give a semantic proof using Kripke structures, and give several conditions on rewrite rules for which the completeness theorem holds. Simply adding (propositional) rewrite rules make this theorem harder to prove.

- Finally, we argue that the tableau method we defined is sound with respect to the cut-free intuitionistic sequents modulo (Sec. 5). Soundness is usually proved by semantic arguments, as building a tableau can be viewed as a search for a countermodel. Our approach is thus more technical, but we reap huge benefits: it entails a constructive cut elimination theorem and sheds light on the relation between intuitionistic tableaux and cut-free sequent calculus. To our knowledge, this has never been studied in the intuitionistic frame (even without rewrite rules), although it is a well-known result in classical logic. As final result, we discuss the computational content of such semantic cut-elimination methods and compare it to proof normalization.

\section{Intuitionistic Sequent Calculus Modulo}

Figure 1 shows a representative sample of the rules and the transformation made on the usual sequent inferences, reformulated to account for the fact that we now manipulate equivalent formulas modulo a rewrite system $(\mathcal{R})$ on terms and atomic propositions. The full calculus can be found in [7]. In this work, $\mathcal{R}$ is 
supposed to be terminating and confluent. When $\mathcal{R}$ is empty, we get back the usual $L J$.

$$
\begin{gathered}
\frac{\Gamma \vdash_{\mathcal{R}} P}{P \vdash_{\mathcal{R}} Q} \text { axiom if } P \equiv_{\mathcal{R}} Q \quad \frac{\Gamma, Q \vdash_{\mathcal{R}} S}{\Gamma, R \vdash_{\mathcal{R}} S} \Rightarrow \text {-l if } R \equiv_{\mathcal{R}}(P \Rightarrow Q) \\
\frac{\Gamma, P \vdash_{\mathcal{R}} Q}{\Gamma \vdash_{\mathcal{R}} R} \Rightarrow \text {-r if } R \equiv_{\mathcal{R}}(P \Rightarrow Q) \quad \frac{\Gamma, P \vdash_{\mathcal{R}} S \quad \Gamma \vdash_{\mathcal{R}} Q}{\Gamma \vdash_{\mathcal{R}} S} \text { cut if } P \equiv_{\mathcal{R}} Q
\end{gathered}
$$

Fig. 1: Some rules of LJ modulo

A cut-free derivation of a sequent is denoted $\Gamma \vdash_{\mathcal{R}}^{*} P$. The semantics put behind these rules is a modified version of Kripke semantics, where negation is expressed in a positive way.

Definition 1 (modified Kripke structures). A modified Kripke structure $\mathcal{K}$ is a quadruple $\langle K, \leq, D, \Vdash\rangle$ such that:

- $K$ is a non-empty set (the worlds), partially ordered by $\leq$.

- $D$ (the domain) is a monotonous function over $K$ : if $\alpha \leq \beta$ then $D_{\alpha} \subseteq D_{\beta}$

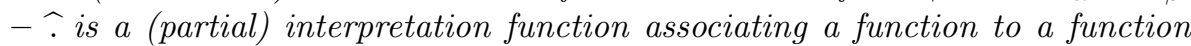
symbol $f$ and a world: $\hat{f}: K \mapsto D_{\alpha}^{m} \mapsto D_{\alpha}$, such that when $\alpha \leq \beta$, $\hat{f}(\alpha)\left(a_{1}, \ldots, a_{n}\right)=\hat{f}(\beta)\left(a_{1}, \ldots, a_{n}\right)$ on $D_{\alpha}$. ( $f$ in $\beta$ extends $f$ in $\left.\alpha\right)$.

- Predicate symbols are interpreted syntactically, which is enough for the scope of this paper.

- The interpretation $|t|_{\sigma}^{\alpha}$ of a term $t$ in $D_{\alpha}$, under a substitution $\sigma$ is defined by induction on $t$ as usual.

- $\Vdash$ is a relation between worlds $\alpha$ and propositions, modulo a substitution $\sigma$ mapping variables to elements of $D_{\alpha}$. It satisfies:

1. Monotonicity on atoms. For any atomic predicate $A\left(x_{1}, \ldots, x_{n}\right)$, any worlds $\beta \geq \alpha$ and terms $t_{1}, \ldots, t_{n}: \alpha \Vdash_{\sigma} A\left(t_{1}, \ldots, t_{n}\right)$ implies $\beta \Vdash_{\sigma} A\left(t_{1}, \ldots, t_{n}\right)$.

2. $\alpha \Vdash_{\sigma} A \vee B$ iff $\alpha \Vdash_{\sigma} A$ or $\alpha \Vdash_{\sigma} B$.

3. $\alpha \Vdash_{\sigma} A \wedge B$ iff $\alpha \Vdash_{\sigma} A$ and $\alpha \Vdash_{\sigma} B$.

4. $\alpha \Vdash_{\sigma} A \Rightarrow B$ iff for any $\beta \geq \alpha, \beta \Vdash_{\sigma} A$ implies $\beta \Vdash_{\sigma} B$.

5. $\alpha \Vdash_{\sigma} \neg A$ iff for any $\beta \geq \alpha, \beta \Vdash_{\sigma} A$ implies $\beta \Vdash_{\sigma} \perp$ (denoted $\left.\beta \nVdash_{\sigma} A\right)$.

6. $\alpha \Vdash_{\sigma} \exists x A$ iff there exists an element $a \in D(\alpha)$ such that $\alpha \Vdash_{\sigma+\langle a / x\rangle} A$.

7. $\alpha \Vdash_{\sigma} \forall x A$ iff for any $\beta \geq \alpha$, for any element $a \in D(\beta), \beta \Vdash_{\sigma+\langle a / x\rangle} A$.

8. The explosion property: if $\beta \Vdash_{\sigma} \perp$ then for any $\alpha \in K$, any proposition $P$, any substitution $\theta, \alpha \Vdash P$.

A modified Kripke structure $\mathcal{K}$ (for short Kripke structure) such that $\alpha \Vdash \perp$ is called improper.

The positive treatment of the negation is essential for constructivity. As we do not have to consider proper Kripke structures, we avoid the use of König's lemma to identify an infinite branch. Our definition is inspired by Veldman's 
[12] and Friedman's [11], but it is perhaps closer to Krivine's classical one ([8]), as we add only one Kripke structure to the usual definition (the improper one).

Our Kripke structures are also constrained to validate rewrite rules: for any world $p \in K$, any formulas $P \equiv_{\mathcal{R}} Q, p \Vdash P$ iff $p \Vdash Q$. This is equivalent ([6]) to considering two formulas $A \rightarrow P$ where $A$ is atomic and $P$ the one-step reduct of $A$ and forcing that for any world $p \in K, p \Vdash A$ iff $p \Vdash P$.

\section{Intuitionistic Predicate Tableaux Modulo}

Proof-search methods for deduction modulo have been developed using both resolution [4] and tableaux [1] for first-order classical logic. We present here a simple ground tableau method for intuitionistic deduction modulo. A tableau is at heart an attempt to define a model of the formulas at its root. Working with Kripke structures forces us to build worlds, therefore a truth statement in a tableau will be a truth statement in a particular world.

Any branch represents a possible model. A closed branch represents a contradiction in the attempt to define a particular model (the one laying on this branch), thus it is improper. If all the branches can be closed, the root formulas should be provable (Sec. 5). The systematic tableau generation of Sec. 4 corresponds to the systematic search of a model.

$A \downarrow$ stands for the normalization of the formula $A$ with respect to the rewrite rules of $\mathcal{R}$ (supposed to be terminating and confluent). We keep the basic expansion rules of [9] in Figure 2, working on statements representing signed forced formulas at some world $p$ (identical Kripke world, hence partially ordered by $\geq$ ). A statement $T p \Vdash P$ (resp. $F p \Vdash P$ ) should be read as the commitment to set $P$ forced (resp. unforced) at world $p . P$ is unforced at world $p$ means that if $P$ is forced, then $\perp$ should also be forced (the Kripke structure we try to define is improper). Notice that we use the same forcing symbol $\Vdash$ when we deal with Kripke structures and that nothing is said about the partial order $\geq$ on worlds. When the sign of a forcing statement is irrelevant, $B$ is a shortcut for " $T$ or $F$ ".

We extend intuitionistic tableaux to use term and propositional rewriting. Expansion rules should be read as follows: when the premise formula is anywhere in the considered path (i.e. not necessarily the leaf), the tableau tree can be expanded with the consequence formula(s). Branch closure is the usual binary closure: we close a branch if both $T p \Vdash P$ and $F q \Vdash P$ occurs on it for $p \leq q$. A tableau is closed and yields a refutation of the input formula when every branch is closed. We choose to pre-normalize every formula with respect to the rewrite system before entering the tableau construction. Therefore we need to be careful when using any rule handling certain quantifiers. Each time a formula is produce by the positive $\forall$ or the negative $\exists$ quantifier rule, we re-normalize it. In the other two quantifier rules, we need not normalize as the constant is fresh also with respect to rewrite rules.

Figure 2 does not explicate how proofs are constructed with the given rules. As we are mainly interested in the generation of a model, we define a systematic complete search within our tableau modulo framework. 
Definition 2 (Systematic tableau generation). We introduce here the notion of complete systematic tableau. We try to construct a tree representing an intuitionistic variation of a Hintikka set which satisfies Lem. 1 conditions. The construction is similar to those in [3, 9]. Our set of worlds $K$ is made of finite sequences of natural numbers, ordered by the prefix ordering. For any world, we define by induction a base set $\mathcal{D}(p)$ :

$-\mathcal{D}(\emptyset)$ is the language constructed over $\mathcal{L}_{0} \cup C_{\emptyset}, \mathcal{L}_{0}$ is the ground terms of the first-order language ;

- if $q$ is the concatenation of the sequence $p$ and the natural number $k(p * k)$, we define $\mathcal{D}(q)$ as the language constructed over $\mathcal{D}(p) \cup C_{q}$, where we have countably many disjoint sets $C_{p}$ of fresh constants for each finite sequence $p$

In each of those sets, we define an enumeration of the terms. We also define an enumeration of the pairs $(p, t)$ where $p$ is a world and $t \in \mathcal{D}(p)$. The tableau is then constructed step by step by expanding each time the least unused node on it (the ordering is based on depth and left-to-right precedences). Let $\pi$ the path from the root to this node. We detail some cases among the most significant:

- If on the path $\pi$ we have two contradictory statements, then close all the branches containing this path.

- Tp $\forall x P(x):$ Let $(q, t)$ the least pair such that $q \geq p, t \in \mathcal{D}(q)$, q occurs on $\pi$, and the statement $T q \Vdash P(t) \downarrow$ does not appear on $\pi$. Attach at each leaf of the branches having $\pi$ as an initial segment the two statements $\{\mathrm{Tq} \Vdash$ $P(t) \downarrow, T p \Vdash \forall x P(x)\}$. We keep an unused copy of $T p \Vdash \forall x P(x)$ in order to go on (later) in the enumeration of the terms and worlds.

- Fp $\forall x P(x)$. Let $k$ the least number such that $p * k$ does not occur on any branch having $\pi$ as initial segment to $F p \Vdash \forall x P(x)$. It is incomparable with any world, but $p$. Let also $c \in C_{q}$ be a fresh constant. Attach at each leaf of the branches extending $\pi$ the statement $F q \Vdash P(c)$.

\begin{tabular}{|c|c|c|c|}
\hline$T p \Vdash A \vee B$ & $F p \Vdash A \wedge B$ & $T p \Vdash A \wedge B$ & $F p \Vdash A \vee B$ \\
\hline$T p \Vdash A \mid T p \Vdash B$ & $F p \Vdash A \mid F p \Vdash B$ & $T p \Vdash A, T p \Vdash B$ & $F p \Vdash A, F p \Vdash B$ \\
\hline$T p \Vdash A \Rightarrow B$ & $F p \Vdash A \Rightarrow B$ & $T p \Vdash \neg A$ & $F p \Vdash \neg A$ \\
\hline $\begin{array}{l}F p^{\prime} \Vdash A, T p^{\prime} \Vdash B \\
\quad \text { for any } p^{\prime} \geq p\end{array}$ & $\begin{array}{l}T p^{\prime} \Vdash A, F p^{\prime} \Vdash B \\
\text { for some new } p^{\prime} \geq p\end{array}$ & $\begin{array}{c}F p^{\prime} \Vdash A \\
\text { for any } p^{\prime} \geq p\end{array}$ & $\begin{array}{c}\frac{T p^{\prime} \Vdash A}{\text { for some new } p^{\prime} \geq p}\end{array}$ \\
\hline$T p \Vdash \exists x P(x)$ & $F p \Vdash \exists x P(x)$ & $T p \Vdash \forall x P(x)$ & $F p \Vdash \forall x P(x)$ \\
\hline $\begin{array}{l}T p \Vdash P(c) \\
\text { for some new } c\end{array}$ & $\begin{array}{l}F p \Vdash P(t) \downarrow \\
\text { for any } t\end{array}$ & $\begin{array}{l}T p^{\prime} \Vdash P(t) \downarrow \\
\text { for any } p^{\prime} \geq p \\
\quad \text { and any } t\end{array}$ & $\begin{array}{c}F p^{\prime} \Vdash P(c) \\
\text { for some new } p^{\prime} \geq p \\
\text { and some new } c\end{array}$ \\
\hline
\end{tabular}

Fig. 2: Rules for intuitionistic predicate tableaux modulo

From Def. 1, we adopt the convention that on a closed branch, any statement appears (since the Kripke structure will be improper). The tableau construction of Def. 2 satisfies the following lemma: 
Lemma 1. Let $\pi$ be a branch of a completely developed tableau by Def. 2. Then:

- if $B p \Vdash P$ appears, then $B p \Vdash P \downarrow$ appears.

- if $T p \Vdash A \wedge B$ (resp. $F p \Vdash A \vee B$ ) appears, then $T p \Vdash A$ and $T p \Vdash B$ (resp. $F p \Vdash A$ and $F p \Vdash B)$ appears.

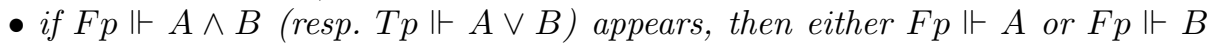
(resp. Tp $\Vdash A$ or $T p \Vdash B)$ appears.

- if $T p \Vdash \neg P$ (resp. Tp $\Vdash A \Rightarrow B$ ) and a world $p^{\prime} \geq p$ appear then $F p^{\prime} \Vdash P$ (resp. $F p^{\prime} \Vdash A$ or $\left.T p^{\prime} \Vdash B\right)$ appears

- if $F p \Vdash \neg P$ (resp. $F p \Vdash A \Rightarrow B$ ) appears then for some world $p^{\prime} \geq p, T p^{\prime} \Vdash P$ (resp. $T p^{\prime} \Vdash A$ and $F p^{\prime} \Vdash B$ ) appears.

- if $F p \Vdash \exists x P(x)$ appears then for every $t \in \mathcal{D}(p)$, Tp $\Vdash P(t) \downarrow$ appears.

- if $T p \Vdash \exists x P(x)$ appears then for some fresh constant $c \in \mathcal{D}(p), P(c)$ appears.

- if $F p \Vdash \forall x P(x)$ appears then for some world $p^{\prime} \geq p$ and some fresh constant $c \in D\left(p^{\prime}\right), T p^{\prime} \Vdash P(c)$ appears.

- if $T p \Vdash \forall x P(x)$ and a world $p^{\prime} \geq p$ appear on the branch, then for every term $t \in D\left(p^{\prime}\right), T p^{\prime} \Vdash P([x:=t] \downarrow$ appears.

Proof. If we define $\tau=\lim \tau_{n}$ the tableau generated by Definition 2, all nodes are used, and we have enumerated all the worlds $q \geq p$ and all the terms of $\mathcal{D}(q)$ (for the $\forall$ statements), no matter if the branch considered is closed or not, from the convention we adopted.

Remark: This lemma proves that an open branch is the exact intuitionistic counterpart of a Hintikka set (in the same way, a Schütte's semi-valuation). But we cannot identify an open branch.

\section{Completeness}

The extended liberality of $L J_{\text {mod }}$ rules entails a harder completeness proof, as the constructed Kripke structure must also be a model of the rewrite rules.

Theorem 1 (Completeness of the tableau method). Let $\mathcal{R}$ be a rewrite system verifying one of the conditions below, $\Gamma$ be a set of propositions, $P$ be a proposition. If for any node $\alpha$ of any Kripke structure $\mathcal{K}, \alpha \Vdash \Gamma$ implies $\alpha \Vdash P$, then any branch of the complete systematic tableau for $T \emptyset \Vdash \Gamma, F \emptyset \Vdash Q$ is closed.

Without rewrite rules, it suffices to set our model from a complete branch of the tableau according to the statements over atoms, and extend it "naturally" to compound formulas. But we have here to ensure that the built Kripke structure is a model of the rewrite rules. We detail such an extension in the following sections, describing large classes of rewrite systems, and the completeness proofs associated.

Moreover, our proofs differ from the usual tableau completeness proof, since we do not consider open branches anymore. We use instead the following plan: from a branch of the completely developped tableau, we define a Kripke structure then we prove that it agrees with the statements on the branch and that it is a 
model of $\mathcal{R}$. We get as result $\emptyset \Vdash \Gamma$, and by hypothesis $\emptyset \Vdash P$. But we also get $(\emptyset \Vdash P) \Rightarrow(\emptyset \Vdash \perp)$, hence the Kripke structure is improper, and we shall meet on the branch $T p \Vdash \perp$ for some $p$. So the branch is closed.

\subsection{An order condition}

It is shown in [6] how to build a model for an order condition. We again give the construction (the Kripke structure built is rather different), but no proof since they are almost the same. We consider a well-founded order $\prec$ such that if $P \rightarrow Q$ then $Q \prec P$ and if $A$ is a subformula of $B$ then $A \prec B$. Given a branch, we define the Kripke structure $\mathcal{K}=\langle K, \leq, D, \Vdash\rangle$ :

$-K=\{p$ sequences of integers $\}$. It is ordered by the order used in the tableau construction.

- $\mathcal{D}(p)$ is the set of closed terms appearing in all the forcing statements involving the world $p$, or a world $q \leq p$.

- The forcing relation $\Vdash$ is defined by induction on the size of formulas. For normal atomic formulas we let $q \Vdash A$ iff $T p \Vdash A$ appears on the branch for some $p \leq q$. We extend this forcing relation to non atomic formulas according to the definition of a Kripke structure. There is three non standard cases: we let $p \Vdash \neg P$ if for any $q \geq p$ we do not have $q \Vdash P$. If $A$ is a non-normal atom, we set $p \Vdash A$ iff $p \Vdash A \downarrow$. At last if we meet $T p \Vdash \perp$ on the branch, then we add $q \Vdash P$ for any $q$ and $P$.

This definition is well-founded as $\prec$ is well-founded. It obviously defines a Kripke structure. We now prove a result that would not be needed with the usual definition of Kripke structures:

Lemma 2. If the Kripke structure is improper, then the branch is closed.

Proof. We can have $p \Vdash \perp$ for two reasons. The first is if the statement $T p \Vdash \perp$ appears. Thus the branch is closed. The second reason is if we have both $p \Vdash P$ and $(p \Vdash P) \Rightarrow(p \Vdash \perp)$, for some world $p$ and formula $P$. The second statement can only be derived from $q \Vdash \neg P$ for some $q \leq p$, but this is never set - since $p \Vdash P$ - unless we already know that $p \Vdash \perp$.

The constructed Kripke structure agrees with the branch: if $T p \Vdash P$ appears on the branch, then $p \Vdash P$. And if $F p \Vdash P$ appears on the branch then $p \nVdash P$. This is obvious for normal atomic formulas, and extended by induction on $\prec$. The case analysis is slightly different, since we now interpret $p \nVdash P$ as "if $p \Vdash P$ then $p \Vdash \perp$ ". We detail some cases below.

- if $F p \Vdash A$ appears, with $A$ a normal atom. Then $p \Vdash A$ is set in the Kripke structure only if it is improper.

- if $F p \Vdash \neg P$ appears. From the tableau construction, we have below in the branch the statement $T p * k \Vdash P$ for some new world $p * k$ - remember that we choose the convention that if $q \Vdash \perp$ appears, then any statement appear. By induction hypothesis, $p * k \Vdash P$. If $p \Vdash \neg P$ then by monotonicity $p * k \Vdash \neg P$ and the Kripke structure is improper. 
Moreover, the Kripke structure is a model of the rewrite rules (by induction on the w.f.o. $\prec)$. We then have: $\emptyset \Vdash \Gamma, \emptyset \nVdash Q$, and $\mathcal{K}$ is a Kripke model of $\mathcal{R}$.

\subsection{A positivity condition}

We present a new condition on propositional rewrite rules. We suppose that if $P \rightarrow Q \in \mathcal{R}$, all atomic predicates occurring in $Q$ occur positively (under an even number of negations/right implication positions). If this condition is fulfilled, we first need to saturate the branch, in order to decide the truth value of as many formulas as possible. We define the saturation process following the definition of a Kripke structure. We enumerate the pairs $(p, P)$ where $p \in K$ and $P$ is a formula over the language $\mathcal{D}(p)$ and add the following statements to the branch:

$-B p \Vdash P$ if $B p \Vdash P \downarrow$ appears.

$-T p \Vdash P($ resp. $F p \Vdash P$ ) if $T q \Vdash P$ (resp. $F q \Vdash P$ ) appears for $q \leq p$ (resp. $q \geq p)$. Truth propagates upwards and falsity downwards, from Def. 1 . .

$-T p \Vdash P \wedge Q$ (resp. $F p \Vdash P \wedge Q)$ if $T p \Vdash P$ and $T p \Vdash Q$ (resp. either $F p \Vdash P$ or $F p \Vdash Q)$ appear.

$-T p \Vdash \neg P$ (resp. $F p \Vdash \neg P$ ) if for any $q \geq p, F q \Vdash P$ (resp. for some $q \geq p$, $T q \Vdash P)$ appears.

- Tp $\forall x P(x)$ (resp. $F p \Vdash \forall x P(x)$ ) if for any $q \geq p$, any term $t \in \mathcal{D}(q)$, $q \Vdash P(t) \downarrow$ (resp. for some $q \geq p$ and some $t \in \mathcal{D}(q), F q \Vdash P(t) \downarrow$ appears.

The steps of this completion process satisfy the conditions that two opposite forcing statements $T p \Vdash P$ and $F q \Vdash P$ for some $q \geq p$ appear only if the branch was already closed, and that Lem. 1 remains valid.

Since the number of formulas having an interpretation increases at each iteration of this process, this operation has a least fixpoint that we take as the branch in the rest of this section.

Lemma 3. • If Tp $\Vdash P$ appears, then for any $q \geq p, T q \Vdash P$ appears.

- If Fp $\Vdash P$ appears, then for any $q \leq p, F q \Vdash P$ appears.

- If $P \equiv_{\mathcal{R}} Q$ and $B p \Vdash P$ appears, then $B p \Vdash Q$ appears.

- The new branch is closed iff the original one is closed.

- The new branch verifies lemma 1

Proof. The completion process entails the two first claims and we have just proved the two last ones. The third one stands because $B p \Vdash P$ appears only if $B p \Vdash P \downarrow$ does (which is proved by induction on the size of $P$ ).

This process is necessary to define a Kripke structure. We need to know as much as possible about every formula. It is really absolutely necessary for Lem. 5. This is the intuitionistic counterpart of Schütte partial valuation, since it satisfies more than Lem. 1. For the formulas, we go not only top-down but also bottom-up. For instance: $F p \Vdash \forall x P(x)$ appears iff for some world $p^{\prime} \geq p$ and some fresh constant $c \in D\left(p^{\prime}\right), T p^{\prime} \Vdash P(c) \downarrow$ appears. The only difference with usual partial valuations is that we could be in a degenerated case. However, 
the valuation is not yet total (some formulas can be left uninterpreted), and we still have no model. So we build the Kripke structure $\mathcal{K}=\langle K, \leq, D, \Vdash\rangle$ as in Sec. 4.1 except that the forcing relation $\Vdash$ is defined by induction on the size of formulas. For every atomic predicate (over the language $\mathcal{D}(q)$ ) we let $q \Vdash A$ if $T q \Vdash A$ appears on the branch. If $F p \Vdash A$ does not appear we also let $p \Vdash A$. We extend this forcing relation to non atomic formulas as before. This model is trivially a Kripke structure. We now prove that $\mathcal{K}$ agrees with the branch:

Lemma 4. If a statement $T p \Vdash P$ (resp.Fp $\Vdash P$ ) appears on the branch, then $p \Vdash P($ resp. $p \nVdash P)$ in the Kripke structure $\mathcal{K}$.

Proof. By induction on the structure of $P$. The base case (atomic) is trivial from the definition. Other cases are immediate as the branch satisfies Lem. 1.

As the Kripke structure agrees with the branch, $\emptyset \Vdash \Gamma$ and $\emptyset \nVdash P$. We now need to show that the Kripke structure is a model of $\mathcal{R}$. We know (Lem. 4) that if $A \rightarrow P$ and $P \downarrow=A \downarrow$ appear in the branch as $B p \Vdash A \downarrow$, then all three formulas $(A, P, P \downarrow)$ share the same forcing relation with $p$. But what if $P \downarrow$ does not appear? Recall then that the rewrite system is positive. Hence $P$ is positive. Let us prove the following lemma:

Lemma 5. Let $P^{+}$be a positive formula and $Q^{-}$be a negative formula (i.e. $\neg Q$ is positive) defined over $\mathcal{D}(p)$. If $B p \Vdash P^{+}$(resp. $B p \Vdash Q^{-}$) does not appear (whether $B=T$ or $B=F)$ in the branch, then $p \Vdash P+\left(\right.$ resp. $\left.p \nVdash Q^{-}\right)$.

Proof. We suppose that no statement $T p \Vdash \perp$ appear in the branch, otherwise $B p \Vdash R$ appear for any $p$ and $R$. Thereforein the (proper) Kripke structure defined $p \nVdash P$ means in particular that we do not have $p \Vdash P$. We proceed by induction on the structure of $P$ and $Q$ and detail only some key cases. If $P$ is an atom, even non normal, then it is positive, and in the constructed Kripke structure, $p \Vdash P$.

If $P^{+}=A^{+} \vee B^{+}$, then since $T p \Vdash P^{+}$does not appear, neither $T p \Vdash A$ nor $T p \Vdash B$ appears. Otherwise $T p \Vdash P$ would have been set by the saturation process. Similarly, either $F p \Vdash A$ or $F p \Vdash B$ does not appear. Suppose the first statement does not appear, then we apply the induction hypothesis to $A$ and get that $p \Vdash A$, therefore $p \Vdash P$. Now if $P^{-}=A^{-} \vee B^{-}$, we have the same results. We have to prove $p \nVdash A$ and $p \nVdash B$. There are two cases: if $F p \Vdash A$ appears, conclude by Lemma 4 otherwise use the induction hypothesis.

If $P=\forall x R^{+}(x)$, let $q \geq p$ be a world and $t \in \mathcal{D}(q)$. $F p \Vdash P^{+}$does not appear, hence no statement $F q \Vdash R(t)$ appear (otherwise $F p \Vdash P$ would have been set by the saturation process). If $T q \Vdash R(t)$ appears, $q \Vdash R(t)$ by Lem. 4. Otherwise $q \Vdash R(t)$ by the induction hypothesis. Therefore, by the Kripke structure definition, $p \Vdash \forall x R^{+}(x)$. If $Q=\forall x R^{-}(x)$ then similarly there is at least one world $q \geq p$ and one term $t \in \mathcal{D}(q)$ for which $T q \Vdash R(t)$ does not appear. If $F q \Vdash R(t)$ appears, we apply Lem. 4 , otherwise we use the induction hypothesis. In both cases, $q \nVdash R(t)$. Thus, by the Kripke structure definition, $p \nVdash \forall x R^{-}(x)$. The other connectors are treated in exactly the same way. 
Now let $A$ be an atom, $p$ a world, and $A \rightarrow P$. If $A$ appears in a statement $B p \Vdash A$, then $B p \Vdash P$ (by Lem. 3) and by Lem. $4 A$ and $P$ have the same interpretation. Otherwise, since $P$ is positive by hypothesis, $p \Vdash P$. And $p \Vdash A$ by definition. Anyway, the rewrite rules are valid in $\mathcal{K}$ which is thus a model.

\subsection{Mixing the two conditions}

Consider two rewrite systems $\mathcal{R}_{>}$and $\mathcal{R}_{+}$. Under the confluence and termination of $\mathcal{R}=\mathcal{R}_{>} \cup \mathcal{R}_{+}$and the condition that $\mathcal{R}_{+}$is right-normal for $\mathcal{R}_{>}$, we are able to prove completeness of the tableau method:

Definition 3 (Right normality). Let two rewrite systems $\mathcal{R}^{\prime}$ and $\mathcal{R}$. $\mathcal{R}^{\prime}$ is right normal for $\mathcal{R}$ if, for any propositional rule $l \rightarrow r \in \mathcal{R}^{\prime}$, all the instances of atoms of $r$ by $\mathcal{R}$-normal substitutions $\sigma$ are in normal form for $\mathcal{R}$.

This condition has never been studied before. The model is built as follows: given a branch, saturate it as in Section 4.2 and define the model by induction on the well-founded order. We interpret non $\mathcal{R}_{+}$-normal atoms exactly as in Section 4.2. The Kripke structure $\mathcal{K}$ agrees as before with the branch and is a model of $\mathcal{R}_{>}$Both claims are proved by induction over the well-founded order $>$. Furthermore, $\mathcal{K}$ is also a model of $\mathcal{R}_{+}$.

Lemma 6. Let $P^{+}$be a positive formula and $Q^{-}$be a negative formula defined over $\mathcal{D}(p)$. Suppose that all instances (by $R$-normal substitutions) of atoms from $P, Q$ are normal for $\mathcal{R}_{>}$.

If $B p \Vdash P^{+}$(resp. Bp $\left.\Vdash Q^{-}\right)$does not appear (whether $B=T$ or $B=F$ ) in the branch, then $p \Vdash P+$ (resp. $\left.p \nVdash Q^{-}\right)$.

Proof. By induction on the formula structure, as lemma 5. Notice that we cannot apply the rewrite rules of $\mathcal{R}_{>}$.

We then can conclude that any $P \rightarrow Q \in \mathcal{R}_{+}$is valid in the Kripke structure.

\subsection{On computational content}

We exhibit a result that will be important in the discussion of the relations between constructive semantic cut elimination and proof normalization. This rewrite rule is already discussed in [7], in a nonconstructive frame. Consider this rewrite system where $A$ is any atomic formula, and $y \simeq z$ stands for $\forall x(y \in x \Rightarrow$ $z \in x)$ :

$$
R \in R \rightarrow \forall y(y \simeq R \Rightarrow(y \in R \Rightarrow(A \Rightarrow A)))
$$

Theorem 2. The tableau modulo method for this rewrite system is complete.

Proof. Given a branch, define the Kripke structure $\mathcal{K}$ as in Section 4.2: it agrees with this branch (proved as in Section 4.2). If the Kripke structure is improper, it means that the branch is closed. Moreover the rewrite rule 1 is valid. Indeed, the formula $R \in R \Leftrightarrow \forall y(y \simeq R \Rightarrow(y \in R \Rightarrow(A \Rightarrow A)))$ is always forced at any node of any Kripke structure (it is an intuitionistic tautology). This completeness proof leads to a cut elimination theorem for this rewrite system. 


\section{Soundness}

We now must prove the soundness of the tableau method w.r.t. cut-free $L J_{\text {mod }}$. In classical logic, it is common knowledge that a ground tableau proof corresponds to a cut-free proof of the sequent calculus. In the intuitionistic case, it is not obvious since a tableau proof corresponds to a sequent with multiple conclusions, understood as a disjunction of formulas, altough sequent calculus has at most one right member. Hence, the soundness of intuitionistic tableaux is always proved with respect to Kripke structures. [3] attempts a syntactic soundness proof but some details seem rather unclear ( $\vee$ case). For that, we first state some definitions, building upon those of [3].

Definition 4. Let $p$ be a world. We define the sets $T_{p}(\pi)=\{P \mid T q \Vdash P \in \pi$ for some $q \leq p$ and $F_{p}(\pi)=\{P \mid F p \Vdash P \in \pi\}$ Let $\bigvee S$ holds for the disjunction of some elements of $S$. A path $\pi$ is consistent if for any world $p, T_{p}(\pi) \nvdash_{\mathcal{R}}^{*} \bigvee F_{p}(\pi)$

Remark: A forcing statement belongs to $\pi$ means that this very statement appears on the path $\pi$. Notice that $T_{p}(\pi)$ contains all true formulas at any world below $p$. On the other hand, $F_{p}(\pi)$ contains the false formulas only at world $p$. This is due to the Kripke structure definition: an unforced formula at $p$ can be forced in a future world, whereas truth is a commitment for all the future worlds. The major difference between our definition and the one of [3] is the definition of consistency of a path.

Associativity of the $\vee$ connector would be a triviality using the cut rule but needs here a proof.

Lemma 7. Let $A, B, C$ be formulas and $\Gamma$ be a set of formulas. If we have a proof $\theta$ of $\Gamma \vdash_{\mathcal{R}}^{*} A \vee(B \vee C)$ then we can construct a proof $\theta^{\prime}$ of $\Gamma \vdash_{\mathcal{R}}^{*}(A \vee B) \vee C$.

Proof. The proof proceeds by induction on $\theta$. We need a stronger induction hypothesis: $\theta$ is a proof of $\Gamma \vdash_{\mathcal{R}}^{*} P$ where $P$ can either be $A \vee(B \vee C)$ or $B \vee C$.

If the last rule is axiom, then replace it by the proof of $P \vdash_{\mathcal{R}}^{*}(A \vee B) \vee C$. If the last rule is $\vee$-r, we get a proof of $\Gamma \vdash_{\mathcal{R}}^{*} A, \Gamma \vdash_{\mathcal{R}}^{*} B, \Gamma \vdash_{\mathcal{R}}^{*} C$, or $\Gamma \vdash_{\mathcal{R}}^{*} B \vee C$. In the first three cases, plug the two $\vee$-r rules for a proof of $\Gamma \vdash_{\mathcal{R}}^{*}(A \vee B) \vee C$. In the last one, apply the induction hypothesis to $\Gamma \vdash_{\mathcal{R}}^{*} B \vee C$. Otherwise, apply the induction hypothesis to the premise(s) (unless $P$ becomes erased) and apply the same rule on $\Gamma \vdash_{\mathcal{R}}^{*}(A \vee B) \vee C$.

The commutativity of $\vee$ is immediate (switching premises). Hence, we now note $\bigvee S$ the disjunction of some subset of $S$, disregarding order and parenthesis. We also can weaken the conclusion, adding $\vee$-r rules to get the disjunction of the whole $S$. The following lemma "strengthens" some rules of the sequent calculus, allowing multiple right propositions. Such properties are trivial with the cut rule. As we want a cut elimination theorem, we need more elaborate proofs.

Lemma 8. Let $A, B, C$ be formulas and $\Gamma_{1}, \Gamma_{2}$ be sets of formulas. From proofs of $\Gamma_{1} \vdash_{\mathcal{R}}^{*} A \vee C$ and $\Gamma_{2} \vdash_{\mathcal{R}}^{*} B \vee C$ (resp. $\Gamma_{1} \vdash_{\mathcal{R}}^{*} P(t) \vee C$, resp. $\Gamma_{1}, B \vdash_{\mathcal{R}}^{*} C$ and $\Gamma_{2} \vdash_{\mathcal{R}}^{*} A \vee C$ ) we can construct a proof of $\Gamma_{1}, \Gamma_{2} \vdash_{\mathcal{R}}^{*}(A \wedge B) \vee C$ (resp. $\Gamma_{1} \vdash_{\mathcal{R}}^{*}(\exists x P(x)) \vee C$, resp. $\left.\Gamma_{1}, \Gamma_{2}, A \Rightarrow B \vdash_{\mathcal{R}}^{*} C\right)$ 
Proof. We focus on the first part of the lemma. The other parts are proved using the same pattern. We construct bottom-up from the two proofs $\pi_{1}$ and $\pi_{2}$ of $\Gamma_{1} \vdash_{\mathcal{R}}^{*} A \vee C$ and $\Gamma_{2} \vdash_{\mathcal{R}}^{*} B \vee C$ a proof of the sequent $\Gamma_{1}, \Gamma_{2} \vdash_{\mathcal{R}}^{*}(A \wedge B) \vee C$.

The idea is simple: first, include a copy of $\pi_{1}$, using $\Gamma_{1}$ then, at the leaves of $\pi_{1}$, when necessary, take a copy of $\pi_{2}$, using $\Gamma_{2}$ (unchanged by the first induction on $\left.\pi_{1}\right)$. Let us detail a bit. We construct a proof of $\Gamma_{1}, \Gamma_{2} \vdash_{\mathcal{R}}^{*}(A \wedge B) \vee C$ by induction on $\pi_{1}$. If the first rule is:

- a rule with $\Gamma_{1}$ as an active formula, apply the induction hypothesis to the premise(s) and then apply the same rule. For instance, for the $\Rightarrow$-l rule:

$$
\frac{\frac{\pi_{1}^{\prime}}{\Gamma_{1}, Q \vdash_{\mathcal{R}}^{*} A \vee C} \frac{\pi_{1}^{\prime \prime}}{\Gamma_{1} \vdash_{\mathcal{R}}^{*} P}}{\Gamma_{1}, P \Rightarrow Q \vdash_{\mathcal{R}}^{*} A \vee C}
$$

we apply the induction hypothesis to $\pi_{1}^{\prime}$, get a proof $\pi^{\prime}$ of $\Gamma_{1}, Q, \Gamma_{2} \vdash_{\mathcal{R}}^{*}$ $(A \wedge B) \vee C$, and we then apply the $\Rightarrow$-l rule:

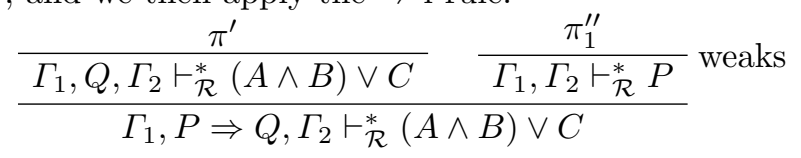

- a right weakening (on $A \vee C$ ). We instead weaken on $(A \wedge B) \vee C$ and add left weakenings to introduce $\Gamma_{2}$. We get a proof of $\Gamma_{1}, \Gamma_{2} \vdash_{\mathcal{R}}^{*}(A \wedge B) \vee C$.

- an axiom rule $\left(\mathcal{A}_{1}\right)$ or a $\vee$-r rule $\left(\mathcal{V}_{1}\right)$. This is the most interesting case. We stop the induction on $\pi_{1}$ and initiate an induction on $\pi_{2}$. As usual, we rename the fresh constants of $\pi_{2}$ in order for them to be fresh for $\Gamma_{1}$. If the first rule is:

- a left rule $r$. Apply $r$ the the proof(s) obtained by induction hypothesis.

- a right weakening. Similar as in the induction on $\pi_{1}$.

- an axiom $\left(\mathcal{A}_{2}\right)$ or a $\vee$-r rule $\left(\mathcal{V}_{2}\right)$. We have four cases. Consider the case $\mathcal{A}_{1}, \mathcal{A}_{2}$ first. We have $P_{A \vee C} \in \Gamma_{1}$ and $P_{B \vee C} \in \Gamma_{2}$ with $P_{A \vee C} \equiv_{\mathcal{R}} A \vee C$ and $P_{B \vee C} \equiv_{\mathcal{R}} B \vee C$. It is trivial to construct a proof of $P_{A \vee C}, P_{B \vee C} \vdash_{\mathcal{R}}^{*}$ $(A \wedge B) \vee C$.

Consider the $\mathcal{V}_{1}$ (similarly $\mathcal{V}_{2}$ ) case. There are two subcases. If the premise is a proof $\pi_{1}^{\prime}$ of $\Gamma_{1} \vdash_{\mathcal{R}}^{*} C^{\prime}$ with $C^{\prime} \equiv_{\mathcal{R}} C$, construct the following proof (ignoring $\pi_{2}$ ):

$$
\frac{\frac{\pi_{1}^{\prime}}{\Gamma_{1}, \Gamma_{2} \vdash_{\mathcal{R}}^{*} C^{\prime}} \text { weaks }}{\Gamma_{1}, \Gamma_{2} \vdash_{\mathcal{R}}^{*}(A \wedge B) \vee C} \vee \text {-right }
$$

Otherwise the premise is a proof $\pi_{1}^{\prime}$ of $\Gamma_{1} \vdash_{\mathcal{R}}^{*} A^{\prime}$ with $A^{\prime} \equiv_{\mathcal{R}} A$. If we have $\mathcal{V}_{2}$ and the premise is a proof $\Gamma_{2} \vdash_{\mathcal{R}}^{*} C^{\prime}$, we construct the above proof, switching indexes 1 and 2. Otherwise, it is a proof of $\Gamma_{2} \vdash_{\mathcal{R}}^{*} B^{\prime}$ with $B^{\prime} \equiv_{\mathcal{R}} B$, and we construct the proof:

$$
\frac{\frac{\pi_{2}^{\prime}}{\Gamma_{1}, \Gamma_{2} \vdash_{\mathcal{R}}^{*} B^{\prime}} \text { weaks } \frac{\pi_{1}^{\prime}}{\Gamma_{1}, \Gamma_{2} \vdash_{\mathcal{R}}^{*} A^{\prime}}}{\frac{\Gamma_{1}, B^{\prime} \vdash_{\mathcal{R}}^{*}(A \wedge B)}{\Gamma_{1}, \Gamma_{2} \vdash_{\mathcal{R}}^{*}(A \wedge B) \vee C} \vee-\mathrm{r}} \text { weaks }
$$


If we have $\mathcal{A}_{2}$ we construct the proof:

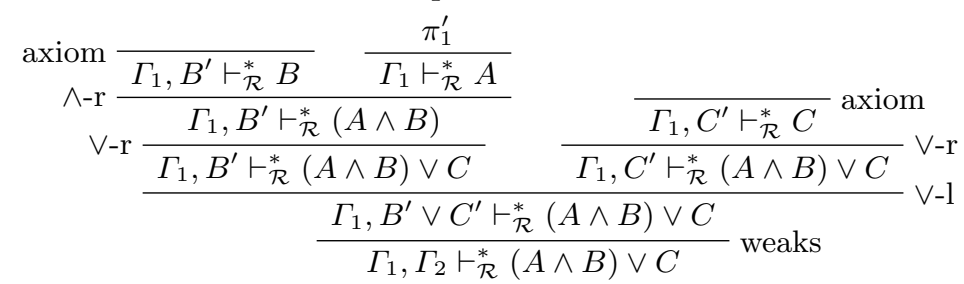

where $B^{\prime} \equiv_{\mathcal{R}} B, C^{\prime} \equiv_{\mathcal{R}} C$ and $B^{\prime} \vee C^{\prime}$ is the formula used in $\mathcal{A}_{2}$. It is a disjunction because $\mathcal{R}$ is confluent and left members are atomic, so main connectors of two equivalent compound formulas are the same $([7,6])$.

The treatment of $\exists$ needs only one induction. $\Rightarrow$ as well: we need an induction on the proof of $\Gamma_{2} \vdash_{\mathcal{R}}^{*} A \vee C\left(\Gamma_{1}, B \vdash_{\mathcal{R}}^{*} C\right.$ contains no disjunction $)$.

Note. This result has to be compared with the LB sequent calculus [15], where these very rules are allowed. However, soundness of LB is proved semantically. Our result seems then to be new.

In the sequel, we use Lem. 8 with $\Gamma_{1}=\Gamma_{2}=\Gamma$. We get a proof of $\Gamma, \Gamma \vdash$ $(A \wedge B) \vee C$ contracted as a proof of $\Gamma \vdash_{\mathcal{R}}^{*}(A \wedge B) \vee C$. We are now ready to prove soundness of the intuitionistic tableau construction with respect to cut-free sequent calculus.

Theorem 3 (Syntactic cut-free soundness of tableaux). If $\Gamma \nvdash_{\mathcal{R}}^{*} P$ then there is a consistent path $\pi$ in the systematic complete tableau developement of $T \emptyset \Vdash \Gamma \downarrow, F \emptyset \Vdash P \downarrow$.

Remark: The contraposite of this theorem has exactly the same proof, complicated by some uninteresting additional cases.

Proof. We show that if $\pi$ is a consistent branch in a partially developed tableau, the method of Sec. 3 extends it (at some step) in at least one consistent path.

The root of the tableau is consistent: having $\Gamma \vdash_{\mathcal{R}}^{*} P$ is the same as having $\Gamma \downarrow \vdash_{\mathcal{R}}^{*} P \downarrow$. This is a classical result of deduction modulo (see for instance [4, 6, $7])$. Now let $B p \Vdash P$ the least unused statement in the tableau developement appearing on $\pi$ ( and $P$ is normal by construction). If $B p \Vdash P$ is:

$-T p \Vdash Q \wedge R, \pi$ is extended following the rules of figure 2 with $T p \Vdash Q$ and $T p \Vdash R$. If the new path is inconsistent, the added statement must be involved, and we have a proof of $T_{p}\left(\pi^{\prime}\right) \vdash_{\mathcal{R}}^{*} F_{p}\left(\pi^{\prime}\right)$ But $T_{p}\left(\pi^{\prime}\right)=T_{p}(\pi) \cup$ $\{Q, R\}$ and $F_{p}\left(\pi^{\prime}\right)=F_{p}(\pi)$. We apply $\wedge$-l and obtain a proof of $T_{p}(\pi), P \vdash_{\mathcal{R}}^{*}$ $F_{p}(\pi)$ contradicting $P \in T_{p}(\pi)$.

- Fp $\Vdash Q \wedge R, \pi$ is extended with two paths $\pi_{0}$ and $\pi_{1}$. If both new paths are inconsistent, we get the two proofs $T_{p}(\pi) \vdash_{\mathcal{R}}^{*} Q \vee \bigvee F_{p}(\pi)$ and $T_{p}(\pi) \vdash_{\mathcal{R}}^{*}$ $R \vee \bigvee F_{p}(\pi)$ with $T_{p}(\pi)=T_{p}\left(\pi_{0}\right)=T_{p}\left(\pi_{1}\right), F_{p}\left(\pi_{0}\right)=F_{p}(\pi) \cup\{Q\}$ and $F_{p}\left(\pi_{1}\right)=F_{p}(\pi) \cup\{R\}$. Potentially weakening (Lem. 7 ), we consider both occurrences of $\bigvee F_{p}(\pi)$ to be equal and we apply Lem. 8 to get a proof of $T_{p}(\pi) \vdash_{\mathcal{R}}^{*}(Q \wedge R) \vee \bigvee F_{p}(\pi)$ i.e. a contradiction, since $Q \wedge R \in F_{p}(\pi)$ 
- $T p \Vdash Q \vee R$. If both new paths are inconsistent, combine with $\vee$-l the proofs $T_{p}(\pi), Q \vdash_{\mathcal{R}}^{*} \bigvee F_{p}(\pi)$ and $T_{p}(\pi), R \vdash_{\mathcal{R}}^{*} \bigvee F_{p}(\pi)$ to get a contradiction.

- Fp $\Vdash Q \vee R$. If the new path is inconsistent, we have a proof of $T_{p}(\pi) \vdash_{\mathcal{R}}^{*}$ $(Q \vee R) \vee \bigvee F_{p}(\pi)$ (using Lem. 7). But $Q \vee R \in F_{p}(\pi)$.

- Tp $\Vdash Q \Rightarrow R$, then if both new paths are inconsistent we have proofs of $T_{p^{\prime}}\left(\pi_{0}\right) \vdash_{\mathcal{R}}^{*} \bigvee F_{p^{\prime}}\left(\pi_{0}\right)$ and $T_{p^{\prime}}\left(\pi_{1}\right) \vdash_{\mathcal{R}}^{*} \bigvee F_{p^{\prime}}\left(\pi_{1}\right)$ since things changing from $\pi$ change at world $p^{\prime}$. By definitions of $T_{p^{\prime}}$ and $F_{p^{\prime}}$, we have proofs of $T_{p^{\prime}}(\pi) \vdash_{\mathcal{R}}^{*} Q \vee \bigvee F_{p^{\prime}}(\pi)$ and $T_{p^{\prime}}(\pi), R \vdash_{\mathcal{R}}^{*} \bigvee F_{p^{\prime}}(\pi)$ By Lem. 8 we get a proof of $T_{p^{\prime}}(\pi), Q \Rightarrow R \vdash_{\mathcal{R}}^{*} \bigvee F_{p^{\prime}}(\pi)$, which contradicts $Q \Rightarrow R \in T_{p^{\prime}}(\pi)$.

- Fp $\Vdash Q \Rightarrow R$. If the new path is inconsistent, we have a proof $\theta$ of $T_{p^{\prime}}\left(\pi^{\prime}\right) \vdash_{\mathcal{R}}^{*}$ $\bigvee F_{p^{\prime}}\left(\pi^{\prime}\right)$. Since $p^{\prime}$ is a new world, comparable only with the $q \leq p$ on $\pi$, $T_{p^{\prime}}\left(\pi^{\prime}\right)=T_{p}(\pi) \cup\{Q\}$ and $F_{p^{\prime}}\left(\pi^{\prime}\right)=\{R\}$. Hence, we can apply the $\Rightarrow$-r rule to $\theta$, and we obtain a proof of $T_{p}(\pi) \vdash_{\mathcal{R}}^{*} Q \Rightarrow R$, yielding the inconsistency of $\pi$ since $Q \Rightarrow R \in F_{p}(\pi)$.

This is extremely important to have no choice for $F_{p^{\prime}}\left(\pi^{\prime}\right)$ but $R$. Exactly at this point the logic gets intuitionistic. Other tableaux methods (like [15]) have also a special treatement of the $\Rightarrow$ and $\forall$ connectors: we need a onemember right side sequent.

$-\neg P$ behaves as $P \Rightarrow \perp$. So both cases are consequences of the previous.

- Tp $\exists x Q(x)$. If the new path is inconsistent, we have a proof of $T_{p}(\pi), Q(c) \vdash_{\mathcal{R}}^{*}$ $\bigvee F_{p}(\pi)$. We apply the $\exists-1$ rule as $c$ is fresh, yielding the inconsistency of $\pi$.

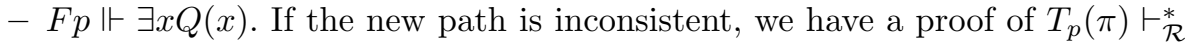
$Q(t) \downarrow \vee \bigvee F_{p}(\pi)$. We transform this proof into a proof of $T_{p}(\pi) \vdash_{\mathcal{R}}^{*} Q(t) \vee$ $\bigvee F_{p}(\pi)$ since $Q(t) \equiv_{\mathcal{R}} Q(t) \downarrow$. Then using lemma 8 we get a proof of: $T_{p}(\pi) \vdash_{\mathcal{R}}^{*} \exists x Q(x) \vee \bigvee F_{p}(\pi)$, thereby contradicting the consistency of $\pi$.

- Tp $\forall x Q(x)$. If the new path is inconsistent, we have a proof of $T_{p^{\prime}}(\pi), Q(t) \downarrow$ $\vdash_{\mathcal{R}}^{*} F_{p^{\prime}}(\pi)$, then converted into a proof of $T_{p^{\prime}}(\pi), Q(t) \vdash_{\mathcal{R}}^{*} F_{p^{\prime}}(\pi)$. Apply the $\forall$-l rule to get $T_{p^{\prime}}(\pi), \forall x Q(x) \vdash_{\mathcal{R}}^{*} F_{p^{\prime}}(\pi)$, and the inconsistency of $\pi$.

- Fp $\forall x Q(x)$. If the new path is inconsistent, we must have a proof of $T_{p^{\prime}}\left(\pi^{\prime}\right) \vdash_{\mathcal{R}}^{*} F_{p^{\prime}}\left(\pi^{\prime}\right)$. As for $F p \Vdash Q \Rightarrow R, p^{\prime}$ is a new world, comparable only with $p$. So, we have in fact a proof of $T_{p}(\pi) \vdash_{\mathcal{R}}^{*} Q(c)$. We apply the $\forall$-r rule, since $c$ is fresh. This yields the inconsistency of $\pi$.

We have established that a closed tableau is a cut-free proof of $L J_{\text {mod }}$. This result is new, even in $L J$. The combination of the soundness theorem of sequent calculus w.r.t. modified Kripke structures, Th. 1 and Th. 3 yields a constructive semantic cut elimination theorem, holding for the conditions seen in Sec. 4:

Theorem 4 (Cut elimination for $L J_{\text {mod }}$ ). If $\Gamma \vdash_{\mathcal{R}} P$ then $\Gamma \vdash_{\mathcal{R}}^{*} P$.

\section{Conclusion and further work}

We have formulated a simple tableau method for intuitionistic logic modulo and proved its completeness and syntactic soundness in order to to show that the computational content our semantic cut elimination theorem is actually a tableau method. Moreover, in [7], it is proven that the rewrite system 1 does not 
possess the proof normalization property: any attempt to normalize the proof (with a cut on $R \in R$ ) of $\vdash_{\mathcal{R}} A \Rightarrow A$ can only fail. We can semantically eliminate this cut, because we have the semantical information that $A \Rightarrow A$ is a tautology. The proof normalization method does not own this information. In this case, the semantic analysis is sharper, and it shows the gap between both methods. The link with normalization by evaluation methods, for instance [2] where a Kripke-style framework is presented, seems a promising field of investigations. The tableau method itself could be much improved with a better handling of the rewrite steps (instead of normalizing - which includes many possibly unnecessary computing). Then we could deal with quantifiers more efficiently : free-variable tableaux based upon $[14,15]$ or the introduction of Skolem symbols (more tricky in intuionistic logic, see $[10,13]$ ) would indeed improve efficiency.

\section{References}

[1] Richard Bonichon. Tamed: A tableau method for deduction modulo. In IJCAR, pages 445-459, 2004.

[2] Catarina Coquand. From semantic to rules: a machine assisted analysis. In CSL, 1993.

[3] Mary De Marco and James Lipton. Completeness and cut elimination in Church's intuitionistic theory of types. Journal of Logic and Computation, 15:821-854, December 2005.

[4] Gilles Dowek, Thérèse Hardin, and Claude Kirchner. Theorem proving modulo. Journal of Automated Reasoning, (31):33-72, 2003.

[5] Gilles Dowek and Benjamin Werner. Proof normalization modulo. The Journal of Symbolic Logic, 68(4):1289-1316, December 2003.

[6] Olivier Hermant. Méthodes Sémantiques en Déduction Modulo. PhD thesis, Université Paris 7 - Denis Diderot, 2005.

[7] Olivier Hermant. Semantic cut elimination in the intuitionistic sequent calculus. Typed Lambda-Calculi and Applications, pages 221-233, 2005.

[8] Jean-Louis Krivine. Une preuve formelle et intuitionniste du théorème de complétude de la logique classique. The Bulletin of Symbolic Logic, 2:405-421, 1996.

[9] Anil Nerode and Richard A. Shore. Logic for Applications. Springer-Verlag, 1993.

[10] Natarajan Shankar. Proof search in the intuitionistic sequent calculus. In $C A D E$, pages 522-536, 1992.

[11] Anne S. Troelstra and Dirk Van Dalen. Constructivism in Mathematics, volume 2. North Holland, 1988.

[12] Wim Veldman. An intuitionistic completeness theorem for intuitionistic predicate logic. Journal of Symbolic Logic, 41:159-166, 1976.

[13] Andrei Voronkov. Proof-search in intuitionistic logic based on constraint satisfaction. In TABLEAUX, pages 312-329, 1996.

[14] Arild Waaler. Handbook of Automated Reasoning, volume II, chapter Connection in Nonclassical Logics. North Holland, 2001.

[15] Arild Waaler and Lincoln Wallen. Handbook of Tableau Methods, chapter Tableaux for Intutionistic Logics, pages 255-296. Kluwer Academic Publishers, 1999. 